\title{
Spontaneous pneumomediastinum in early postbirth period (clinical observation)
}

\begin{abstract}
Summary
The article describes a clinical case of spontaneous pneumomediastinum in a 20 -year-old patient in the early postpartum period. The diagnosis was made during the first hour of the postpartum period based on the patient's complaints and confirmed by instrumental research methods. Analysis of the presented clinical situation suggested a possible cause of the resulting pathology, which are the inferiority of the lung tissue due to severe pneumonia and the presence of indirect signs of connective tissue dysplasia.
\end{abstract}

Keywords: pneumomediastinum, postpartum period, connective tissue dysplasia, pneumonia
Volume 8 Issue 4 - 2020

\author{
Kukharchyk YV, Dovnar RI, Kukharchyk IV, \\ Hussein Abdimahad Abdullahi \\ Grodno State Medical University, Belarus
}

Correspondence: Kukharchyk YV, Department of Obstetrics and Gynecology, Grodno State Medical University, Grodno, Belarus, Tel+375297886617, Email juliakukarchik@mail.ru

Received: July 09, 2020 | Published: August 14, 2020

\section{Introduction}

Pneumomediastinum (mediastinal emphysema) - the presence in the soft tissues of the mediastinum of air or gas coming from the trachea, bronchi, lungs, abdominal cavity, esophagus when they are damaged. Accompanied by chest pains, shortness of breath, subcutaneous emphysema of the neck, face, chest. A significant amount of air in the mediastinum leads to a violation of cardiac activity., ${ }^{1,2}$ It should be noted that in adults, spontaneous pneumomediastinum is more common than in children. Mostly young people under the age of 30 suffer. Among the cases, $76 \%$ are men, $24 \%$ - women. ${ }^{3,4}$ The prevalence of spontaneous emphysema of the mediastinum in puerperas has not been established, in the international literature there are reports of only a study of a small number of cases, or a description of single observations. ${ }^{1,5,6}$

I would like to note that mediastinal emphysema and subcutaneous emphysema can develop in childbirth during the expulsion period as a result of increased bronchial pressure and pulmonary barotrauma due to forced difficult exhalation during attempts, i.e. when an intensified deep breath passes into an arbitrarily restrained exhalation. This leads to trauma to the alveoli, often against the background of inferiority of the lung tissue during pneumosclerosis, diffuse or local emphysema, bronchiectasis, cysts, and respiratory malformations. ${ }^{7,8}$ The available data from experimental studies show that when the alveoli rupture due to an increase in pressure in them, air penetrates into the perialveolar tissue, stratifying it. While maintaining intra-alveolar pressure, air can spread through the interstitial tissue of the lung in the central direction, stratifying the connective tissue bed of vessels and bronchi, and reaches the mediastinum, causing mediastinal emphysema. From the mediastinum, air is distributed into the subcutaneous tissue of the neck, face, front surface of the chest, and shoulder girdle. In addition, air accumulated in the mediastinum can spread downward into retroperitoneal tissues, causing intestinal pneumonosis. The exit of air from the mediastinum is a favorable factor, since otherwise the accumulation of air in the mediastinum under high pressure can compress the vessels passing in the mediastinum and lead to the death of the patient. ${ }^{2,8,9}$ Long attempts are especially dangerous.

Due to the rarity of the disease, nonspecific and scarce clinical symptoms, lack of alertness and sufficient experience among clinicians in relation to this nosology in puerperas, we present our observation of the case of pneumomediastinum in the early postpartum period.

\section{Purpose}

To analyze the features of the clinical picture, diagnosis and treatment of spontaneous pneumomediastinum in the puerperal in the early postpartum period.

\section{Materials and research methods}

We examined a postpartum woman who is hospitalized in the City Clinical Emergency Hospital of Grodno with a diagnosed pneumomediastinum in the early postpartum period. Based on the informed consent of the patient participating in the study, biochemical and instrumental studies were performed.

\section{Research results and discussion}

The pre-pregnant, primiparous R, 20 years old, was admitted to the public health institution "City Clinical Emergency Hospital of Grodno" in 2019 with a diagnosis of "I period of the first urgent birth in the head presentation in the gestational age of 278 days; premature rupture of membranes; minor anomaly of the heart: mitral valve prolapse I degree; euthyroid diffuse goiter of I degree; mild anemia.

The heredity of a woman is not burdened, earlier development was age-appropriate. Obstetric and gynecological history: menarche at 12.5 years, menstruation moderate, painless, established during the first year, lasting 4-6 days, after 28-30 days. Violations of the menstrual cycle is not noted. Last menstruation 04/03/2018. Sexual life since 18 years old, married. Real pregnancy is the first. The woman denies the presence of gynecological diseases. The patient was registered for pregnancy in the antenatal clinic in the city of Grodno in the period of 10-11 weeks. A full clinical and laboratory examination was carried out, consulted by specialists in accordance with the clinical protocols of the Ministry of Health of the Republic of Belarus "Medical observation and medical care for women in obstetrics and gynecology” № 17 dated 02/19/2018.

When performing ultrasound screening studies, deviations from the norm were not detected. The course of this pregnancy was complicated by the threat of termination: at 28-30 weeks, tocolytic therapy was carried out in a hospital. At 39-40 weeks, the patient was admitted to the public health institution "City Clinical Emergency Hospital of Grodno" with a diagnosis of I period of the first emergency birth in the head presentation in the gestational age of 278 days; premature 
rupture of membranes; minor anomaly of the heart: mitral valve prolapse I degree; euthyroid diffuse goiter of I degree; mild anemia.

From the anamnesis it was found that once or twice a year a woman suffers from acute respiratory infections. In childhood, she suffered severe focal pneumonia. Fluorography of the lungs, performed in May 2017, without pathological changes. General condition upon admission is satisfactory. The woman complained of cramping pain in the lower abdomen, amniotic fluid flow at 12 hours and 30 minutes. Anthropometric characteristics: height $158 \mathrm{~cm}$, body weight $60 \mathrm{~kg}$, BMI $24,03 \mathrm{~kg} / \mathrm{m}^{2}$. The physique is asthenic. The skin and visible mucous membranes are clean, peripheral lymph nodes are not enlarged. The mammary glands are soft, the nipples are clean. The vesicular breathing, no wheezing. Heart sounds are rhythmic, heart rate 78 per minute. Blood pressure 115/75-115/80 mm RT. Art. The abdomen is enlarged due to the pregnant uterus, painless on palpation. The position of the fetus is longitudinal; the head is presented, pressed to the entrance to the small pelvis. The fetal heartbeat is clear, rhythmic 148 beats per minute. No swelling.

Gynecological examination data. Visually: the external genitalia are developed correctly; hair growth is of a female type. Inspection using mirrors: the cervix is cylindrical in shape, macroscopically unchanged. Light amniotic fluid is leaking. Vaginal examination: the vagina does not give birth, the cervix is centered, up to $1.5 \mathrm{~cm}$ long. The cervical canal passes two transverse fingers freely. There are no deformities in the pelvis. The cape of the sacrum is not reached. The results of clinical and laboratory studies within the reference values for a given gestational age. ECG data - no pathological changes.

The first period of labor proceeded without complications, antispasmodic and metabolic therapy was carried out. The duration of the first period of labor was 7 hours 25 minutes, the second period - 1 hour, the third period - 15 minutes. A live male child was born weighing $3350 \mathrm{~g}$, height $53 \mathrm{~cm}, 8 / 9$ points according to Apgar. Blood loss was $450 \mathrm{ml}$. One hour after the birth, a feeling of fullness of the neck, face, pain behind the sternum appeared, the voice changed, the tongue increased, speech was disturbed, breathing became difficult, hyperemia of the face, eyelids overhanging the eyeballs appeared. Initially, this condition was regarded as Quincke's edema. Later, upon palpation of the anterior surface of the chest to the eighth rib, arms, neck, face (more to the left), crepitus of the subcutaneous tissue appeared. Shortness of breath began to increase up to 28 respiratory movements per minute, tachycardia up to 104 per minute, hypotension (blood pressure 80-90 / $50 \mathrm{~mm} \mathrm{Hg}$ ). Above all fields of the lungs, weakened breathing was heard. The surgeon on duty was called. Performed X-ray examination (Figure 1).

Diagnosed with spontaneous mediastinal emphysema. On the x-ray of the chest organs (see Figure 1) in the supine position, the transparency of the pulmonary fields is reduced due to the large accumulation of air in the soft tissues of the chest. The mediastinal shadow is located in the middle, the sheets of the mediastinal pleura are "stratified" by an accumulation of air. The heart is bordered by a strip of air. On the radiograph in a lateral projection, the presence of air in the anterior sections of the mediastinum is clearly defined. Conclusion: X-ray picture of mediastinal emphysema, subcutaneous emphysema. Ultrasound imaging of the chest organs is impossible due to the high pneumatization of the subcutaneous tissue, no signs of free fluid in the chest were detected. On the electrocardiogram there is a slight deviation of the electrical axis of the heart to the left, moderate changes in the myocardium of the left ventricle, mainly of metabolic and metabolic nature.

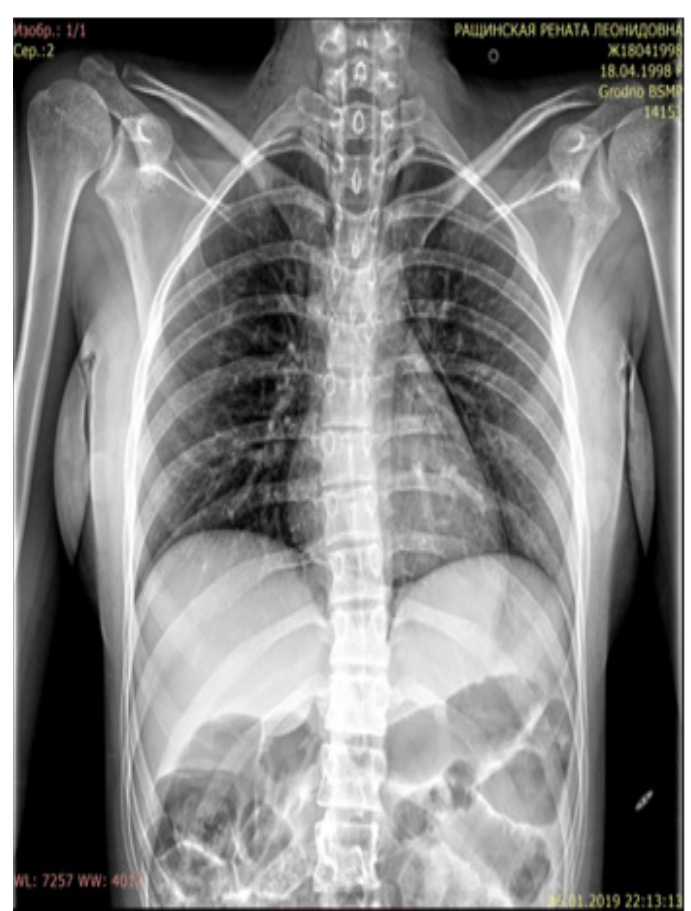

Figure I Chest x-ray of patient R. showing pneumomediastinum.

The patient underwent sedation therapy, drug suppression of the cough reflex, antibiotic therapy. On the second day of the postpartum period, an increase in temperature to $37-37,1{ }^{\circ} \mathrm{C}$ was noted. With dynamic control (January 7, 2019) (Figure 2), a pronounced subcutaneous emphysema of the neck and soft tissues of the chest is preserved on the chest radiograph in direct projection. Pneumomediastinum decreased, but a small amount of air is retained and is well defined on the radiograph in lateral projection. Diagnosis confirmed by thoracic surgeon.

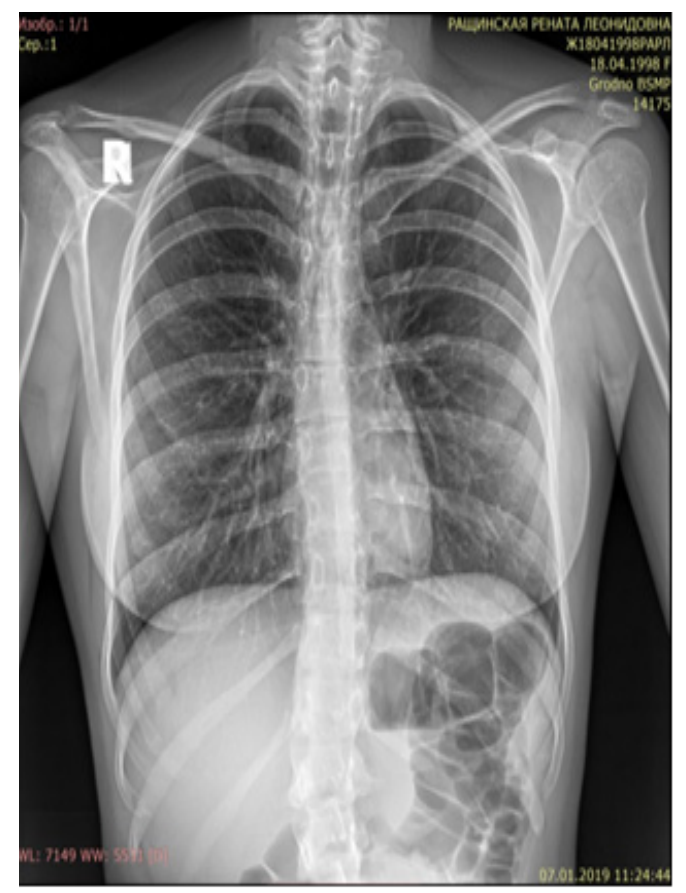

Figure 2 Chest $x$-ray of patient $R$. in direct projection on the first day. 
The clinical signs of pneumomediastinum gradually disappeared, the postpartum period was uneventful and on the 8th day the patient and her child were discharged in a satisfactory condition home. Currently, the patient's condition is satisfactory. Thus pneumomediastinum (mediastinal emphysema) - the presence in the soft tissues of the mediastinum of air or gas coming from the trachea, bronchi, lungs, abdominal cavity, esophagus when they are damaged. Patho-physiologically, the development of pneumomediastinum during spontaneous delivery is linked to the sudden laceration of the alveola due to the increase of intrathoracic and intra-abdominal pressure caused by repeated Valsalva maneuvers.

The main physiological alterations of the respiratory system that occurred during the last part of pregnancy are mainly the consequence of the progestin stimulation of the respiratory drive and consist of a reduction in the functional residual capacity and an increase of about $70 \%$ in alveolar ventilation due to a breathing pattern with augmented respiratory rate and tidal volume. During the second stage of labor, hyperventilation and increase of intra-abdominal pressure caused by repeated Valsalva maneuvers are risk factors for the development of pneumothorax and pneumomediastinum, especially in patients with pre-existent subpleural blebs.

\section{Conclusion}

In the presented clinical case, there was a typical picture of the development of pneumomediastinum and subcutaneous emphysema in the early postpartum period. Perhaps predisposing moments to the development of this pathology in this situation were active laborious activities that contribute to increased intrabronchial pressure and air penetration into the mediastinum against the background of inferiority of lung tissue due to severe pneumonia, as well as connective tissue dysplasia, which, as a rule, accompanies asthenic syndrome. It should be noted that the onset of symptoms of pregnancy-related pneumomediastinum usually develops during labor; however, clinical appearance is often delayed until the postpartum phase. The symptoms chest pains, shortness of breath. Accompanied by chest pains, shortness of breath, dyspnea, cough, palpitations, subcutaneous emphysema of the neck, face, chest. A chest X-ray usually reveals the subcutaneous emphysema, and significant pneumomediastinum. Our patient was primiparous and had regular length of the first and second stages of labor. She delivered with an important pushing effort as she had not any form of analgesia. In addition, Pneumothorax occurs due to a sudden rise in mediastinal pressure with rupture of the parietal mediastinal pleura. As I mentioned above the pathophysiological.

\section{Acknowledgments}

None.

\section{Conflicts of interest}

Authors declare that there is no conflict of interest.

\section{References}

1. Platov II, Moiseev BC. Spontaneous pneumomediastinum. Problems of Tuberculosis. 1998;5:61-62.

2. Hamilton S, Nicholson S, Beattie GJ. Spontaneous oesophageal rupture in the second stage of labor. J Obstet Gynaecol. 2009;23(6):668-673.

3. Abakumov MM, Shamba HL, Danielyan Sh N. Clinic, diagnosis and treatment of mediastinal spontaneous emphysema. Surgery. 2010;10:1721

4. Kvirikhin VT, Migunov KI. Emphysema of the mediastinum in childbirth. Sov Medicine. 1986;8:119-120.

5. Abakumov MM, Shamba HL, Danielyan Sh N. Spontaneous emphysema of the mediastinum. Surgery. 2010;2:47-50.

6. Freixinet J, García F, Rodríguez PM, et al. Spontaneous pneumomediastinum long-term follow-up. Respir Med. 2005;99(9):1160 1163

7. Buchanan GM, Franklin V. Hamman and Boerhaave syndromes diagnostic dilemmas in a patient presenting with Hyperemesis gravidarum: a case report. Scott Med J. 2014;59(4):12-16.

8. Segev T, Alane J, Jacob L, et al. Spontaneous postpartum pneumomediastinum presentation of a rare case and literature review. Obstetrics and Gynecology. 2015;125(2):630-633.

9. Veremchuk JA, Matyushechkina TG, Denisova OS. Pathophysiological mechanisms of obstructive disorders of the ventilation function of the lungs. Klinich Bulletin. 1997;1:66-67. 